http://jmscr.igmpublication.org/home/ ISSN (e)-2347-176x ISSN (p) 2455-0450 crossref DOI: https://dx.doi.org/10.18535/jmscr/v10i1.08

\title{
Epidemiological and Clinical Feature of Newly Diagnosed Childhood Immune Thrombocytopenic Purpura in Qatar
}

\author{
Authors \\ Dr Budoor Kahtan Taha Al-Shammari MD \\ Co Investigators \\ Shabina Khan MD, Rasha Qaqish MD, Yaslam Balfaqih MD, \\ Yasmine Sobeih MD, Najla Ba Shaharil MD \\ Supervised by \\ Dr Ahmad Al-Hammadi, MD, FRCPC \\ Arab Board for Medical Specialties Pediatrics \\ Hamad Medical Corporation in Qatar
}

\begin{abstract}
ITP (Immune thrombocytopenic purpura) is the most common bleeding disorder in childhood. It is usually a self-limiting disorder, and most patients recover spontaneously without serious complication. The clinical features of ITP have remained unchanged over the past few decades but there have been many recent changes in management strategies as evidenced by the new international guidelines on the management of childhood ITP. We wanted to study our pediatric inpatient population in Qatar who were admitted with acute ITP over the last 5 years for their presenting features and the management strategies adopted by our pediatricians. Most of these decisions in our institution are in conjunction with the pediatric hematology team. This study would also serve as baseline data, which could be used to compare changes in trends of management in the future.
\end{abstract}

\section{Introduction}

ITP was previously known as idiopathic thrombocytopenic purpura has now a new term (immune Thrombocytopenia) preserving the widely recognized acronym "ITP" and acknowledges the immune mediated mechanism of the disorder. Immune thrombocytopenia (ITP) of childhood is an immune disease against platelets triggered by many factors leading to low platelet count $<100,000 / \mathrm{microl}$ with normal red and white blood cells count. Triggering factors can be viruses mostly or it can be unknown causes. Most commonly it present with petechie, purpura and bleeding from body orifices due to the low platelet count. It is diagnosed by exclusion of other causes of thrombocytopenia. ITP children have three modalities of treatment steroid, anti Rh, IVIG and spleenectomy reserved for chronic cases.

Although typically it improves spontaneously within three months, a few children will have persistent ITP, which is defined as ITP that lasts more than 3 months but less than 12 months. Approximately 20 percent of affected children 
will go on to have chronic ITP, defined as ongoing thrombocytopenia more than 12 months from presentation.

\section{Review of Literature}

The presenting features of Acute ITP in children has remained unchanged over the past few decades, as evidenced by literature review of previous studies ${ }^{(3)}$ However, with ongoing researches in the field, we definitely have a better understanding of the pathophysiology of ITP in both children and adults. In a large 10 year prospective Canadian study by Jadavji et al, MMR was noted to be linked to the development of acute ITP in children. ${ }^{(4)}$ Likewise, a preceding history of viral illness, allergic reaction or insect bite reported by parents has frequently been elicited in otherwise healthy children presenting with acute ITP $^{(5)}$ Recent researches in this field have supported this, by proving that these seemingly harmless viral or environmental triggers can produce a "pro-inflammatory state" in patients with ITP. ${ }^{(6)}$ We would like to study our pediatric inpatient population who were diagnosed with acute ITP over the last 5 years for any such preceding "triggers" by reviewing any history of antecedent exposure to immunization and viral illnesses, especially those cases where a positive viral PCR was isolated by serological testing of the patient's nasal secretions and blood samples.

Another focus of our study is to review our inpatient population retrospectively for the appropriateness of hospitalization including the platelet count at admission, presence of significant bleeding at presentation and for development of any life threatening bleeding (eg. Intracranial hemorrhage). The risk of intracranial hemorrhage is less than $1 \%$ in patients with acute ITP. ${ }^{(7)}$ Despite this low risk, it is the fear of CNS bleeding, which often prompts physicians to admit these patients for observation. ${ }^{(8)}$ As per both the

American Society of Hematology practices guidelines and their counterparts: the British Guidelines for Management of ITP, hospitalization of patients for observation of intracranial hemorrhage is not warranted ${ }^{(9)}$. Our study will reflect the current trends and criteria followed by our physicians for admitting patients with acute ITP. A study in 2002 on hospitalized pediatric patients with acute ITP concluded that those patients who had "typical" features of acute ITP in terms of their age group (1-10 years), physical findings (no significant hepatosplenomegaly) and laboratory studies (platelet count below 50,000 but otherwise normal blood counts), had a benign course with rapid recovery. (8) By similarly reviewing the epidemiological characteristics of our hospitalized ITP patients in Qatar we may be able to retrospectively define a subset of patients who may not need hospitalization for acute ITP and be good candidates for outpatient management in the future.

\section{Method and Procedure}

This was designed as a retrospective descriptive study. We included previously healthy, newly diagnosed ITP, aged 0 to 14 years, admitted to the pediatric inpatient unit, in Hamad General Hospital, Doha, Qatar from January 2008 to January 2014. Patients with a pre-existing chronic medical illness, including hematological conditions and those that were later diagnosed to have conditions other than ITP, were excluded. Data was collected from the patient's medical records using a specially designed data collection sheet that was used to gather all the relevant patient related details including demographics, presenting signs and symptoms, investigations, treatment and outcome. The study was approved by the Medical Research Center at Hamad Medical Corporation. 

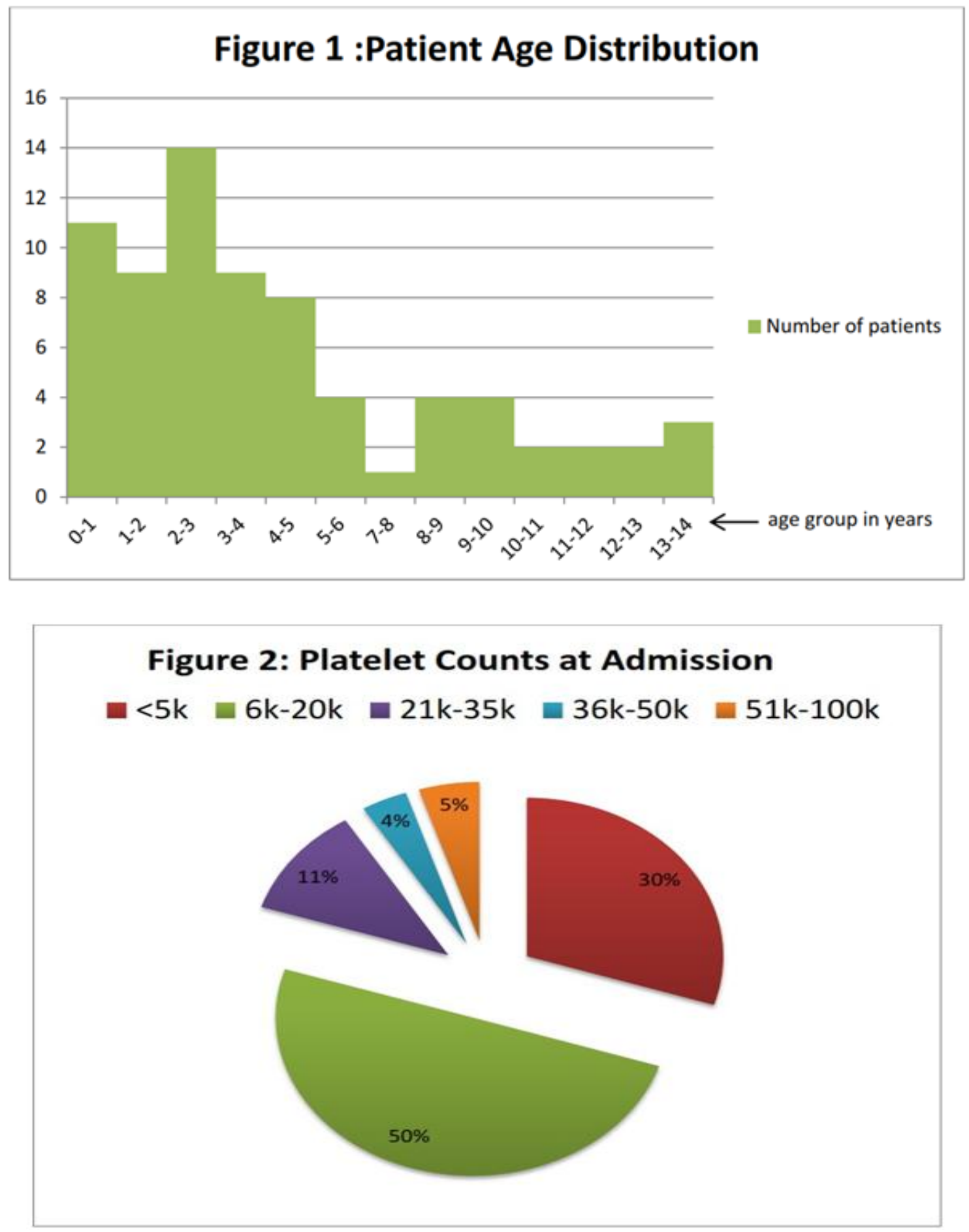

The inclusion criteria of the study is all cases of newly diagnosed ITP admitted to the pediatric inpatient unit in Hamad General Hospital with age less than and equal to 14 years in the last 5 years. The exclusion criteria of the study was :

1. Children older than 14 years

2. Children with known chronic illness e.g. (neurological, cardiac etc)

3. Children with persistent or chronic ITP or with a pre-existing diagnosis of acute ITP at admission
4. Immunodeficient child

5. Congenital hematological abnormalities like hemophilia, G6PD, sickle cell disease, fanconi, thalasemia, VWD disease

6. Other causes of thrombocytopenia like aplastic anemia, HUS, TTP, leukemia, lymphoma.

Regarding the confidentiality that all data collection sheets and data gathered and everything related to the study will be saved on a personal 
computer belonging to Lead PI (Dr Ahmad Alhammadi) and there will be no access of this data to the general population.

\section{Results}

80 patients fulfilled the inclusion criteria with a male to female ratio of $1.1: 1$. (38.3\%) of the patients were in the 2-5 year age group, which reflects the peak incidence of ITP in children as per literature, $(13.8 \%)$ of the patients were below the age of 12 months and (16.3\%) were above the age of $10.34 .3 \%)$ of the patients were Qatari Nationals, while the rest of them were expatriates residing in Qatar.

(40.5\%) reported flu-like symptoms before the presentation of the illness. Most of the parents $(>80 \%)$ denied any history of known hematological disease in their families.

The platelet counts at admission to the pediatric ward were as follows: (80\%) of patients had a platelet count of 20,000 or less, (11.3\%) from 21,000 to 35,000 , while the rest of the patients had platelet counts between 36,000 to 100,000 . The most common clinical features reported were spontaneous sub-cutaneous bruising in $(77.5 \%)$ of patients, followed by oral mucosal petechial rash in $(38.8 \%)$, fever in $(28.8 \%)$, epistaxis in $(16.3 \%)$, gum bleeding in (7.5\%).3 patients needed observation in the pediatric intensive care unit due to life threatening bleeding, one of whom had intracranial bleeding.

The treatment modalities used in our patient population were as follows: Intravenous immunoglobulin (IVIG) alone in (86.3\%), a combination of IVIG and steroids in $(6.3 \%)$ and steroids alone $(1.1 \%)$. Only 5 patients $(6.3 \%)$ were managed by observation alone, 4 of these patients had platelet counts above 20,000 and none had any significant bleeding clinically. The most common IVIG dosing used was 1 gram $/ \mathrm{kg} /$ day for 2 days. (28.7\%) of our study population was prescribed a second dose of IVIG by their treating physician. The vast majority (74.3\%) did not experience any adverse reaction after IVIG treatment. However, (10.8\%) had fever, $(8 \%)$ had vomiting and headache. 2 patients were clinically suspected of aseptic meningitis, post IVIG therapy, based on the treating physician's assessment. In terms of the length of stay in hospital, majority of the children (83.8\%) were hospitalized for 1 to 5 days.

At follow up, (66\%) children had recovered with normal platelet counts within 1 year from the date of diagnosis, while (34\%) had progressed to chronic ITP, which is defined as the persistent thrombocytopenia, lasting beyond 1 year.

\section{Figure 3: Top Five Clinical Features}

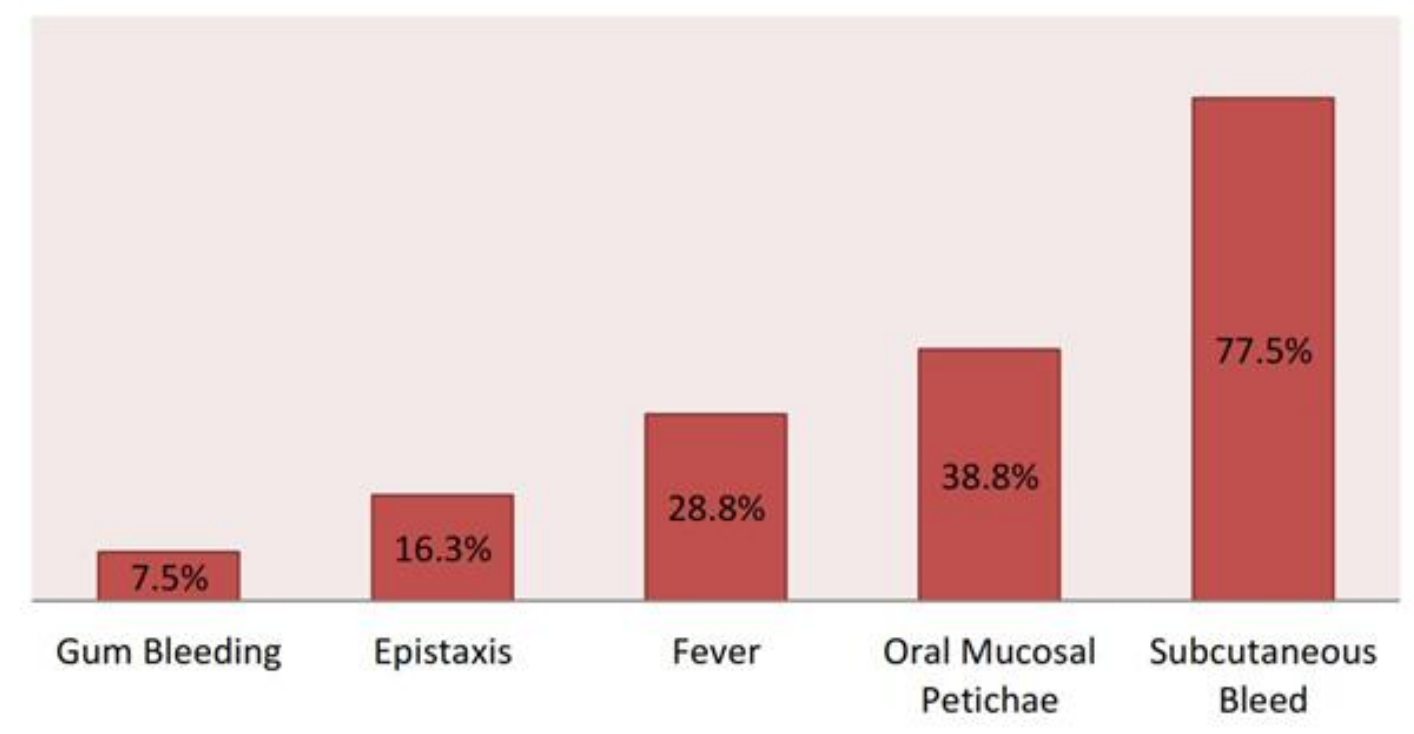




\section{Figure 4: Treatment Modalities}

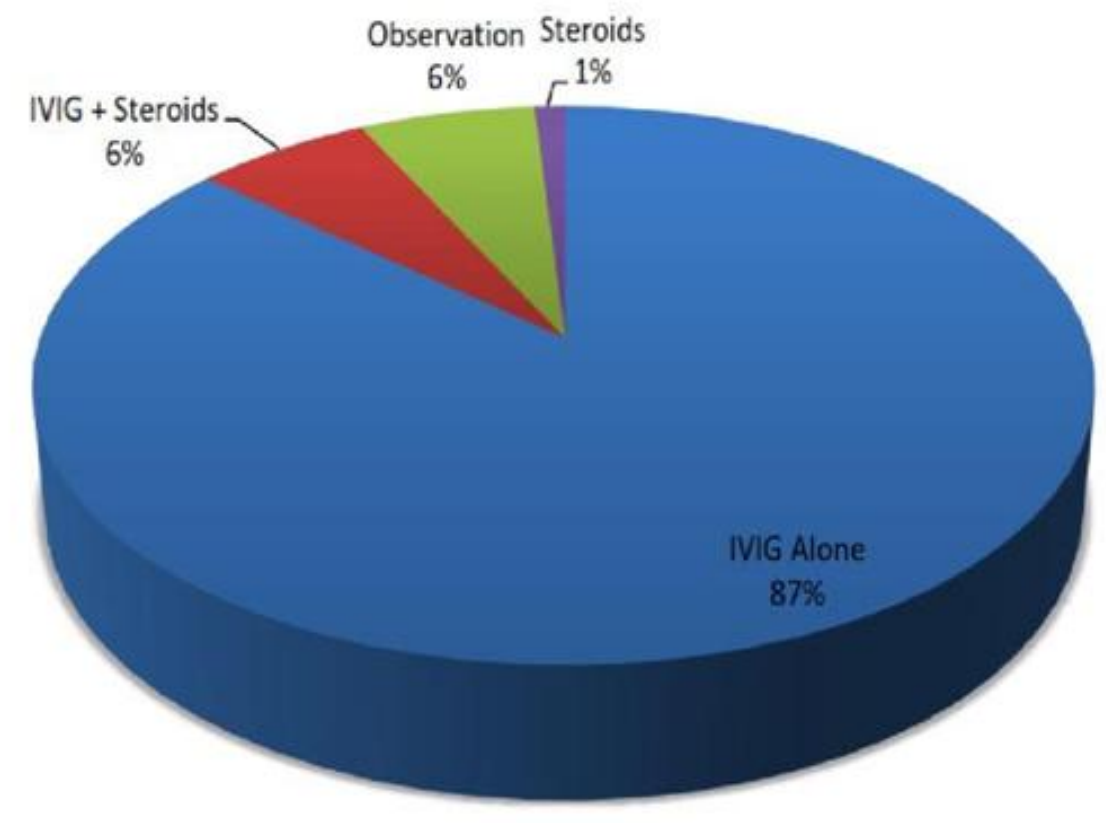

\section{Conclusion}

Our study showed that the clinical features of Acute ITP in Qatar were similar to those reported from various parts of the world. However, the percentage of chronic ITP was higher in our study population than that quoted in literature. Management decisions in our center, like in many centers around the world, were often based on the physician's clinical judgment, rather than the current established international guidelines.

\section{References}

1. Rodeghiero $\mathrm{F}$ et al, Standardization of terminology, definitions and outcome criteria in immune thrombocytopenic purpura of adults and children: report from an international working group. Blood: 2009; 113:2386

2. Management of Immune thrombocytopenic purpura: An update, Rajasekharan et al, Ochsner J. 2012 Fall; 12 (3): 221-227

3. Tarantino M. The treatment of immune thrombocytopenic purpura in children.Curr Hematol Rep. 2006;5:89-94.
4. Jadavji T, Scheifele D, Halperin S. Thrombocytopenia after immunization of Canadian children, 1992 to 2001. Pediatr Infect Dis J. 2003;22:119-122

5. Immune Thrombocytopenic Purpura of Childhood, Diane Nugent, MD, Children's Hospital of Orange County, American Society of Hematology Education Book, Jan 2006 vol 2006 no.1 (97-103)

6. Mouzaki A, Theodoropoulou M, Gianakopoulos I, Vlaha V, Kyrtsonis MC, Maniatis A. Expression patterns of Th1 and Th2 cytokine genes in childhood idiopathic thrombocytopenic purpura (ITP) at presentation and their modulation by intravenous immunoglobulin G (IVIg) treatment: their role in prognosis. Blood.2002;100:1774-1779

7. Lilleyman JS. Intracranial hemorrhage in idiopathic thrombocytopenic purpura.Arch Dis Child. 1994;71:251-3.

8. Hospitalization of children with acute immune thrombocytopenic purpura-is it necessary? Paediatr Child Health. 2002 July-August; 7 (6): 386-389 
9. George JN, Woolf SH, Raskob GE, et al. Idiopathic thrombocytopenic purpura: A practice guideline developed by explicit methods for the American Society of Hematology. Blood. 1996;88:3-40. 